\title{
Controlo da Hipertensão Arterial nos Cuidados de Saúde Primários: Uma Comparação entre Nativos Portugueses e Imigrantes dos Países Africanos de Língua Oficial Portuguesa
}

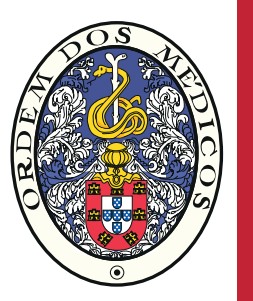

\section{Hypertension Control at the Primary Health Care: A Comparison Among Portuguese Natives and Portuguese Speaking African Coutries Immigrants}

Elisa LOPES $\bigotimes^{1}$, Violeta ALARCÃO ${ }^{1}$, Rui SIMÕES ${ }^{1}$, Milene FERNANDES ${ }^{1,2}$, Verónica GÓMEZ ${ }^{1,3}$, Diana SOUTO ${ }^{1}$, Paulo NOGUEIRA ${ }^{1,4}$, Paulo J. NICOLA ${ }^{1}$, Evangelista ROCHA ${ }^{1}$

Acta Med Port 2016 Mar;29(3):193-204 - http://dx.doi.org/10.20344/amp.6714

RESUMO

Introdução: Em Portugal, a percentagem de hipertensos tratados e controlados é relativamente baixa. Desconhece-se a relação dos determinantes socioeconómicos com o controlo tensional, particularmente nos imigrantes africanos.

Objetivo: Comparar a frequência de controlo nos hipertensos tratados e identificar características associadas à hipertensão tratada não controlada, entre nativos portugueses (caucasianos) e imigrantes dos PALOP (negros).

Material e Métodos: Estudo transversal de hipertensos tratados, com 40-80 anos, aleatorizados dos Cuidados de Saúde Primários da região de Lisboa. Recolheram-se dados sociodemográficos, clínicos e cuidados de saúde por entrevistas estruturadas. Comparou-se a frequência de hipertensos não controlados nos dois grupos, identificando-se fatores relacionados por análise univariada e multivariada.

Resultados: Participaram 786 hipertensos tratados (taxa de participação: $71 \%$ ): 449 nativos e 337 imigrantes. Destes, $46 \%$ tinham a hipertensão controlada. A pressão arterial diastólica foi mais elevada nos imigrantes mais novos. Nos nativos, o não controlo associou-se a: sexo masculino, menor grau de escolaridade, ida aos serviços de urgência e/ou enfermagem e não ida ao médico de família; nos imigrantes, ser solteiro, recorrer ao farmacêutico, número de anos de doença e não adesão intencional à terapêutica.

Discussão: O controlo da hipertensão tratada tem vindo a aumentar nos últimos anos. Nativos e imigrantes diferenciam-se no controlo tensional relativamente à frequência do recurso a consulta do médico de família, e de outros serviços e profissionais de saúde. Estas diferenças não se refletiram em taxas de controlo estatisticamente significativas.

Conclusões: Será necessário definir estratégias para o controlo da hipertensão nos cuidados de saúde primários diferenciadas para os grupos étnicos.

Palavras-chave: Cuidados de Saúde Primários; Factores Socioeconómicos; Grupos Étnicos; Hipertensão/prevenção e controlo; Portugal.

\section{ABSTRACT}

Introduction: In Portugal, the frequency of patient with treated and controlled hypertension is low. It is unknown the relation of socioeconomic determinants with hypertension control, particularly in African immigrants.

Aims: To compare frequency of control in treated hypertension and to identify characteristics associated with uncontrolled and treated hypertension between Portuguese natives (Caucasian) and Portuguese Speaking African Coutries immigrants (black).

Material and Methods: Cross-sectional study of patients with treated hypertension, 40-80 years old, randomized from Primary Health Care of Lisbon Region. We collected sociodemographic, clinical and health care data through structured interviews. We compared the frequency of patients with uncontrolled hypertension, and identified related factors through univariate and multivariate analysis.

Results: In this study participated 786 patients with treated hypertension (participation rate: $71 \%$ ): 449 natives and 337 immigrants. Of these, $46 \%$ had controlled hypertension. Diastolic blood pressure was higher in younger immigrants. Were associated with no control, in natives, male sex, low education, going to emergency and / or nursing services and not looking for the family doctor; on immigrants, being single, using the pharmacist, the number of years of illness and intentional non-adherence.

Discussion: Treated hypertension control has been increasing for last years. Natives and immigrants differ, regarding blood pressure control, relatively to the frequency of family doctor consultation, and resorting to other services and health professionals. These differences didn't reflect in statistically different control rates.

Conclusions: It is needed to define strategies to control hypertension in primary health care specific for ethnic groups.

Keywords: Ethnic Groups; Hypertension/prevention \& control; Portugal; Primary Health Care; Socioeconomic Factors.

\section{INTRODUÇÃO}

A hipertensão arterial (HTA) afeta $42,1 \%$ da população adulta residente em Portugal Continental, dos quais 38,9\%

tomam medicação anti-hipertensiva e, destes, $28,9 \%$ têm a pressão arterial controlada. ${ }^{1}$ Um estudo nos cuidados de

\footnotetext{
1. Unidade de Epidemiologia. Instituto de Medicina Preventiva e Saúde Pública. Faculdade de Medicina. Universidade de Lisboa. Lisboa. Portugal.

2. Eurotrials, Consultores Científicos. Lisboa. Portugal.

3. Departamento de Genética Humana. Instituto Nacional de Saúde Dr. Ricardo Jorge. Lisboa. Portugal.

4. Direç̧ão de Serviços de Informação e Análise. Direcção Geral da Saúde. Lisboa. Portugal.

$\triangle$ Autor correspondente: Elisa Lopes. elisamflopes@gmail.com

Recebido: 16 de junho de 2015 - Aceite: 24 de novembro de 2015 | Copyright @ Ordem dos Médicos 2016
} 
saúde primários (CSP) portugueses descreveu que, dos utentes hipertensos entre 18 e 75 anos, apenas $30,7 \%$ têm a hipertensão tratada e controlada (HTA-TC). ${ }^{2}$ Controlar a HTA, sendo difícil de conseguir na prática clínica, é um desafio relevante porque os hipertensos não controlados têm risco acrescido de morte prematura, de eventos cerebrovasculares e de insuficiência cardíaca. ${ }^{3,4}$ É fundamental aprofundar a capacidade de controlo da HTA em consonância com o preconizado no Programa Nacional para as Doenças Cérebro-Cardiovasculares. ${ }^{5,6}$

Portugal é um país com forte presença e tradição de imigrantes dos Países Africanos de Língua Oficial Portuguesa (PALOP), que constituem um em cada 100 residentes. ${ }^{7}$ As doenças cardiovasculares são mais frequentes nas minorias étnicas ${ }^{8}$, possivelmente devido às condições ambientais a que estiveram expostos nos seus países de origem e estilos de vida menos saudáveis. ${ }^{9}$ Além disso, os imigrantes podem ter problemas de saúde mais cedo e mais frequentemente do que os nativos devido a adversidades sociais e económicas, fatores de stress psicossociais ${ }^{10}$ ou mesmo fatores genéticos e biológicos. ${ }^{11}$ Adicionalmente, os imigrantes podem deparar-se com barreiras linguísticas, socioculturais, económicas e administrativas quando necessitam de recorrer aos serviços de saúde. ${ }^{12,13}$

Estudos americanos ${ }^{13-19}$ e europeus ${ }^{20-23}$ têm demonstrado que os africanos (negros) têm a HTA menos controlada que a população nativa caucasiana. Os fatores que têm sido associados à HTA tratada não controlada (HTA-TnC), tanto na população portuguesa ${ }^{3,24-27}$ como nos africanos (negros), 13,15,17,18,20,22,23 são: sexo masculino, idade, menor escolaridade, não adesão à medicação, obesidade e diabetes. Outros estudos demonstram não haver influência das características sociodemográficas nos imigrantes ${ }^{19,21} \mathrm{e}$ que aqueles que aderem à medicação têm taxas de HTA-TC semelhantes aos nativos, sugerindo que a não adesão pode ser a responsável pelo menor controlo da HTA nos imigrantes. ${ }^{16}$ Outra observação consistente é que os fatores associados à HTA-TnC apresentam gradientes maiores na população negra, com consequente repercussão na ocorrência de doenças cardiovasculares e na necessidade de tratamentos mais intensivos. ${ }^{16,18,19,21}$

Apesar da relevância dada a este assunto noutros países, não conhecemos quais as diferenças no controlo da HTA nos CSP, local de eleição para o seguimento desta doença crónica, entre portugueses e migrantes, duas populações que podem apresentar perfis socioculturais e económicos distintos.

Os objetivos deste estudo são a comparação da frequência de controlo nos hipertensos tratados e a identificação das características associadas à HTA-TnC, entre nativos portugueses e imigrantes dos PALOP, seguidos nos CSP.

\section{MATERIAL E MÉTODOS}

Estudo transversal com hipertensos tratados, imigrantes dos PALOP e nativos portugueses, seguidos nos CSP da Região de Lisboa. Esta análise corresponde à caracteri- zação basal da coorte do estudo DIMATCH-HTA. ${ }^{28}$

Incluímos hipertensos entre 40 e 80 anos, medicados com anti-hipertensivos na altura da entrevista e que tiveram pelo menos uma consulta com o médico de família nos últimos 12 meses.

Considerámos "imigrantes dos PALOP" os indivíduos naturais de um PALOP e que se identificaram como sendo de raça negra, e "nativos" os naturais de Portugal de raça caucasiana. Excluímos os indivíduos retornados (portugueses nascidos em África, caucasianos, que retornaram a Portugal após a revolução de 1974), institucionalizados, sem contato telefónico, dependentes de terceiros para a toma da medicação ou com problemas cognitivos.

Baseámos o processo de amostragem numa abordagem por etapas, aleatorizando a seleção dos Agrupamentos de Centros de Saúde da Região de Lisboa, dos Centros de Saúde/Unidades de Saúde Familiares e dos utentes elegíveis dos médicos com maior proporção de imigrantes.

Entre setembro de 2010 e março de 2011 realizámos contatos telefónicos para recrutamento dos participantes e entrevistas individuais semiestruturadas, maioritariamente nas unidades de saúde, após consentimento informado assinado.

Durante a entrevista, realizámos três medições da pressão arterial, segundo os procedimentos das guidelines for the Management of Arterial Hypertension. ${ }^{29}$ Considerámos indivíduos com HTA-TnC aqueles com medicação anti-hipertensiva e cuja média das três medições foi $\geq 140 \mathrm{mmHg}$ na pressão arterial sistólica (PAS) ou $\geq 90 \mathrm{mmHg}$ na pressão arterial diastólica (PAD).

Calculámos a pressão arterial média (PAM) através da fórmula PAM $=$ PAD + 1/3 * (PAS - PAD). ${ }^{30}$

As características sociodemográficas incluíram idade, sexo, raça e nível socioeconómico da família. Este último foi determinado pela escala de Graffar, considerando as variáveis atividade profissional, escolaridade, fonte de rendimento, tipo de alojamento e características do local de residência, classificando-se em três escalões: alto/médio-alto, médio e médio-baixo/baixo. ${ }^{31}$

A utilização dos cuidados de saúde caracterizou-se pelo sistema de saúde utilizado com mais frequência, se tinha seguro de saúde e pelos serviços de saúde utilizados no último ano para controlar a HTA.

Considerámos como características clínicas: número de anos após o diagnóstico de HTA, ajuda de terceiros para medição da HTA, número diário de fármacos anti-hipertensivos, perímetro abdominal (normal: $<102 \mathrm{~cm}$ nos homens ou $<88 \mathrm{~cm}$ nas mulheres), diagnóstico de diabetes, colesterol elevado e obesidade (índice de massa corporal $\geq 30$ $\mathrm{kg} / \mathrm{m}^{2}$ ).

Avaliámos a adesão à terapêutica nas duas semanas anteriores à entrevista através da escala Medida da Adesão aos Tratamentos, com sete itens (adaptada da escala de Morisky-Green). ${ }^{32}$ Os participantes que responderam "sim" a pelo menos uma questão foram classificados como não aderentes, sendo não aderentes não intencionais (esquecimento ou descuido na toma da medicação $)^{33}$ ou não aderentes intencionais (restantes motivos). ${ }^{34,35}$ 


\section{Análise estatística}

Comparámos os grupos pelos testes Qui-Quadrado para variáveis categóricas e t-Student ou Wilcoxon para variáveis contínuas. Considerámos o nível de significância estatístico $5 \%$ para todos os testes e recorremos ao software $\mathrm{R}$, versão 2.12.2. Nas variáveis categóricas não dicotómicas, quando o teste Qui-Quadrado resultou num valor de $p<0,05$, analisámos gráficos mosaico para identificar qual a categoria que diferia das restantes.

Para o cálculo da percentagem de indivíduos não controlados ajustada para idade e sexo, definimos modelos de regressão logística, específicos de cada grupo. Realizámos uma análise semelhante para calcular os valores médios ajustados de PAS, PAD e PAM através de modelos de regressão linear.

$\mathrm{Na}$ análise univariada dos fatores associados à HTA-
-TnC realizámos modelos de regressão logística obtendo Odds Ratio (OR) e respetivo intervalo de confiança (IC) de $95 \%$. Para as variáveis categóricas calculámos um risco atribuível populacional $[R A P=P e(R R e-1) /[1+\mathrm{Pe}(\mathrm{RRe}-$ 1)], ${ }^{36}$ que avalia o impacto da exposição a um determinado fator no controlo da HTA em função da prevalência $(\mathrm{Pe})$ desse fator na população e da associação (RRe) do fator ao não controlo da HTA.

$\mathrm{Na}$ análise multivariada, construímos modelos de regressão logística hierárquicos a partir das variáveis com valor $p$ inferior a $25 \%{ }^{37}$ na análise univariada. Os modelos consideraram sucessivamente ajustes para as características sociodemográficas, cuidados de saúde, clínicas e adesão à terapêutica, para compreender a influência do efeito sucessivo de cada dimensão no controlo da HTA (considerámos a HTA-TnC como variável dependente). Avaliámos o

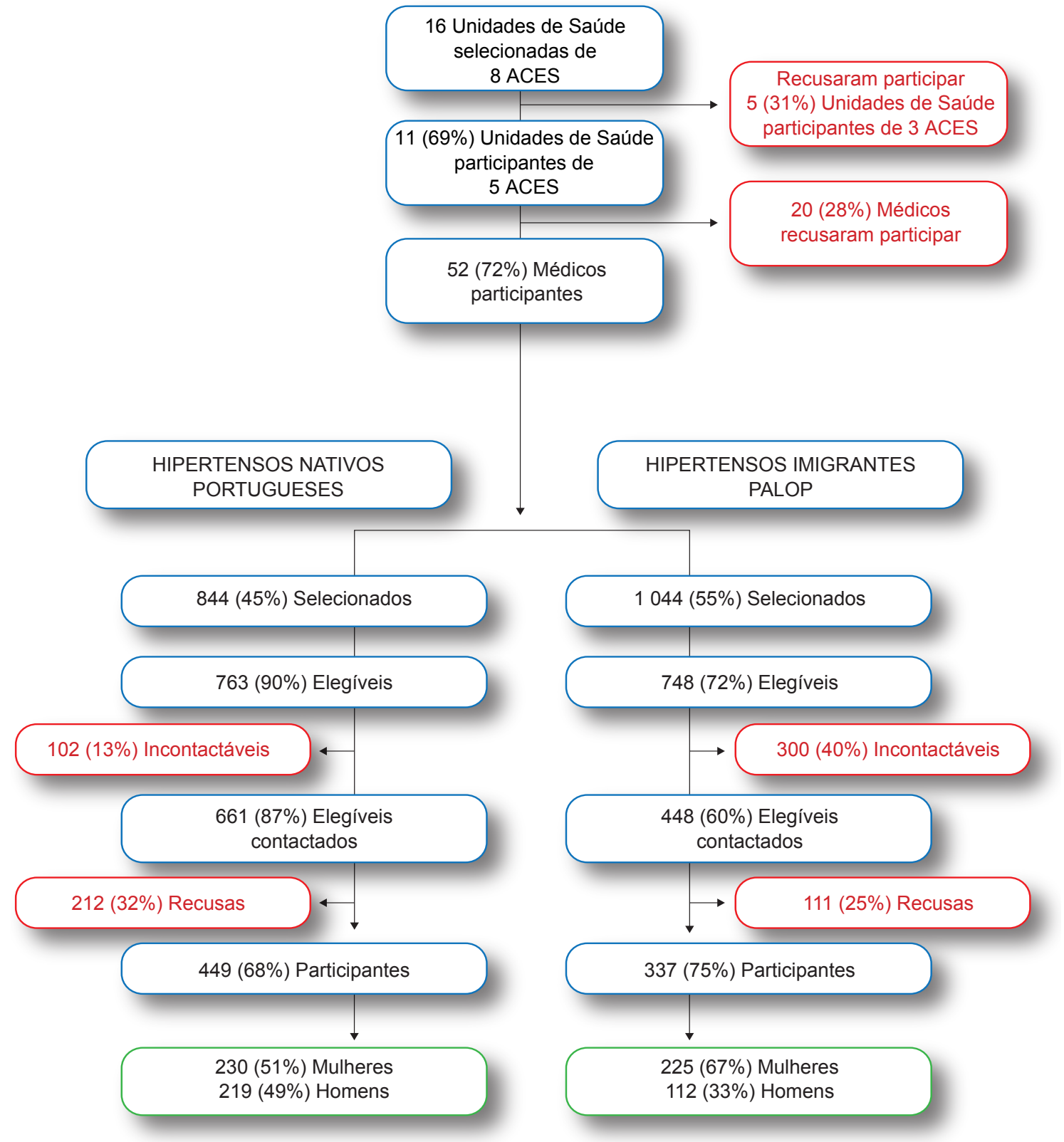

Figura 1 - Recrutamento dos participantes.

ACES: Agrupamentos de Centros de Saúde; PALOP: Países Africanos de Língua Oficial Portuguesa. 
Tabela 1 - Caracterização dos participantes

\begin{tabular}{|c|c|c|c|}
\hline & $\begin{array}{c}\text { Total } \\
(n=786)\end{array}$ & $\begin{array}{l}\text { Nativos } \\
(n=449)\end{array}$ & $\begin{array}{l}\text { Imigrantes dos PALOP } \\
\qquad(n=337)\end{array}$ \\
\hline \multicolumn{4}{|c|}{ Características sociodemográficas } \\
\hline Idade, anos, média \pm dp * & $61,2 \pm 10,2$ & $64,2 \pm 9,1$ & $57,1 \pm 10,1$ \\
\hline Sexo masculino, $\mathbf{n}(\%)$ * & $331(42,1 \%)$ & $219(48,8 \%)$ & $112(33,2 \%)$ \\
\hline \multicolumn{4}{|l|}{ Estado civil, $\mathrm{n}(\%)$} \\
\hline Solteiro/a * & $90(11,5 \%)$ & $19(4,2 \%)$ & $71(21,1 \%)$ \\
\hline Casado/a & $534(67,9 \%)$ & $334(74,4 \%)$ & $200(59,3 \%)$ \\
\hline Divorciado/a & $62(7,9 \%)$ & $38(8,5 \%)$ & $24(7,1 \%)$ \\
\hline Viúvo/a & $99(12,6 \%)$ & $57(12,7 \%)$ & $42(12,5 \%)$ \\
\hline$N^{0}$ de anos escolaridade, média \pm dp & $6,6 \pm 4,3$ & $6,7 \pm 4,1$ & $6,4 \pm 4,5$ \\
\hline $\mathbf{N}^{\circ}$ de anos de residência em Portugal, média $\pm \mathrm{dp}$ & - & - & $25,1 \pm 12,6$ \\
\hline$N^{0}$ pessoas que coabitam, média $\pm d p$ * & $1,9 \pm 1,5$ & $1,4 \pm 1,0$ & $2,6 \pm 1,8$ \\
\hline \multicolumn{4}{|l|}{ Índice Graffar, n (\%) } \\
\hline NSE médio alto / alto & $112(14,9 \%)$ & $67(15,5 \%)$ & $45(14,0 \%)$ \\
\hline NSE médio & $411(54,6 \%)$ & $253(58,7 \%)$ & $158(49,1 \%)$ \\
\hline NSE baixo / médio baixo * & $230(30,5 \%)$ & $111(25,8 \%)$ & $119(36,9 \%)$ \\
\hline \multicolumn{4}{|l|}{ Dificuldade financeira em comprar medicamentos, $\mathbf{n}(\%)$} \\
\hline Não foi nada difícil * & $307(39,9 \%)$ & $230(52,6 \%)$ & $77(23,2 \%)$ \\
\hline Foi um pouco difícil & $240(31,2 \%)$ & $134(30,7 \%)$ & $106(31,9 \%)$ \\
\hline Muito difícil * & $222(28,9 \%)$ & $73(16,7 \%)$ & $149(44,9 \%)$ \\
\hline \multicolumn{4}{|c|}{ Cuidados de saúde } \\
\hline Sem seguro de saúde, $n(\%)$ * & $665(85,1 \%)$ & $365(81,8 \%)$ & $300(89,6 \%)$ \\
\hline Tem ajuda para controlar HTA, n (\%) & $102(13,1 \%)$ & $59(13,3 \%)$ & $43(12,8 \%)$ \\
\hline \multicolumn{4}{|l|}{ Recorreu no último ano para controlar a HTA, n (\%) } \\
\hline Médico de família & $760(97,2 \%)$ & $436(97,8 \%)$ & $324(96,4 \%)$ \\
\hline Especialista no hospital * & $86(11,0 \%)$ & $39(8,7 \%)$ & $47(13,9 \%)$ \\
\hline Serviço de urgência * & $86(11,0 \%)$ & $38(8,5 \%)$ & $48(14,2 \%)$ \\
\hline Farmacêutico * & $227(29,1 \%)$ & $88(19,9 \%)$ & $139(41,4 \%)$ \\
\hline Enfermeiro * & $62(7,9 \%)$ & $27(6,1 \%)$ & $35(10,4 \%)$ \\
\hline \multicolumn{4}{|c|}{ Características clínicas } \\
\hline Anos desde o diagnóstico de HTA, média \pm dp * & $13,3 \pm 10,9$ & $13,9 \pm 10,8$ & $12,6 \pm 10,9$ \\
\hline$N^{\circ}$ fármacos HTA, mediana (IIQ) & $2(1-3)$ & $2(1-3)$ & $2(1-3)$ \\
\hline Obesidade, IMC $\geq 30, \mathrm{n}(\%)$ & $332(49,1 \%)$ & $179(47,6 \%)$ & $153(51,0 \%)$ \\
\hline Diabetes, n (\%) & $212(27,0 \%)$ & $120(26,7 \%)$ & $92(27,3 \%)$ \\
\hline Dislipidemia, n (\%) & $413(54,3 \%)$ & $246(56,0 \%)$ & $167(51,9 \%)$ \\
\hline Perímetro abdominal aumentado, n (\%) & $190(26,8 \%)$ & $111(27,8 \%)$ & $79(25,6 \%)$ \\
\hline
\end{tabular}

* Valor de $p \leq 0,05$, para diferenças nas características entre nativos e imigrantes dos PALOP.

dp: desvio-padrão; HTA: hipertensão arterial; IIQ: intervalo inter-quartílico; IMC: índice de massa corporal; NSE: nível socioeconómico; PALOP: Países Africanos de Língua Oficial Portuguesa. 
poder de descriminação dos modelos finais a partir da área sob a curva ROC (AUC - area under the curve). Considerámos os modelos aceitáveis quando obtívemos curvas ROC com valores superiores a $0,70 .{ }^{37}$

\section{RESULTADOS}

\section{Descrição dos participantes}

Dos 1511 hipertensos tratados elegíveis (763 nativos e 748 imigrantes), não foi possível contatar 26,6\%. Dos 1109 convidados, 786 aceitaram participar [taxa de participação global de 71\%: 68\% nativos dos 661 convidados e $75 \%$ imigrantes dos 448 convidados, $p<0,01$ (Qui-Quadrado)] (Fig. 1). Dos 786 participantes, $449(58,8 \%)$ eram nativos $(51,2 \%$ mulheres) e $337(45,1 \%)$ eram imigrantes $(66,8 \%$ mulheres).

Relativamente às características sociodemográficas (Tabela 1), os nativos distinguiram-se dos imigrantes $(p<$ $0,05)$ pela idade superior $(64,2 \pm 9,1$ anos vs $57,1 \pm 10,1$ anos), predomínio do sexo masculino (48,8\% vs $33,2 \%$ ) e menor dificuldade na compra de medicamentos ("nada difícil": $52,6 \%$ nativos vs $23,2 \%$ imigrantes). Os imigrantes apresentaram, comparando aos nativos $(p<0,05)$, maior frequência de solteiros $(21,1 \%$ vs $4,2 \%)$, coabitantes $(2,6$ $\pm 1,8$ vs 1,4 $\pm 1,0$ ), nível socioeconómico baixo/médio baixo $(36,9 \%$ vs $25,8 \%)$ e maiores dificuldades financeiras na compra de medicamentos ("muito difícil": 44,9\% vs 16,7\%).

Quanto aos cuidados de saúde (Tabela 1), a percentagem de hipertensos que necessitava de ajuda para controlar a HTA era idêntica nos dois grupos (13\%), mas os imigrantes recorreram mais frequentemente ao especialista no hospital (13,9\% vs $8,7 \%)$, ao serviço de urgência $(14,2 \%$ vs $8,5 \%)$, da farmácia $(41,4 \%$ vs $19,9 \%)$ e da enfermagem $(10,4 \%$ vs $6,1 \%)$, sendo todas estas diferenças estatisticamente significativas.

Quanto às características clínicas, os nativos apresentaram, significativamente, maior duração da $\operatorname{HTA}(13,9$ $\pm 10,8$ vs $12,6 \pm 10,9$ anos) (Tabela 1) e maior adesão à medicação anti-hipertensiva (52,7\% vs 34,8\%), enquanto os imigrantes apresentaram maior não adesão intencional
(28,8\% vs $12,2 \%)$ (Tabela 2$).$ Os dois grupos não apresentaram diferenças quanto às restantes variáveis (Tabela 1).

\section{Controlo da Hipertensão Arterial}

Relativamente à frequência de HTA-TC, não verificámos diferenças significativas entre os grupos $(47,2 \%$ nativos vs 45,4\% imigrantes) quando comparados de forma não ajustada (Tabela 2). No entanto, a PAM era significativamente mais elevada nos imigrantes $(106,2 \mathrm{mmHg}$ vs $102,7 \mathrm{mmHg})$, o que se devia à PAD mais elevada $(88,4$ $\mathrm{mmHg}$ vs 83,6 $\mathrm{mmHg}$ ).

Ajustando para diferenças de idade e sexo nos dois grupos, estimamos que menos imigrantes $(47,9 \%$ nativos vs $42,9 \%$ imigrantes) tinham a HTA-TC $(p=0,19)$, e as diferenças ajustadas mantiveram-se relativamente à PAM $(103,0 \mathrm{mmHg}$ vs 106,3 mmHG, $p<0,01)$ e à PAD $(84,5$ $\mathrm{mmHg}$ vs $87,5 \mathrm{mmHg}, p<0,01$ ), e acentuaram-se na PAS (140,3 mm Hg vs 143,9 mmHg, $p=0,02$ ).

Analisada a PAM, PAD e PAS pelos grupos etários (40 64 anos e 65 - 80 anos) e etnias, os imigrantes mais jovens apresentaram significativamente valores mais elevados de PAD $(89,7 \mathrm{mmHg})$, comparando aos nativos do mesmo grupo etário $(86,6 \mathrm{mmHg})$ ou aos mais idosos (imigrantes: 84,5 $\mathrm{mmHg}$; nativos: $80,9 \mathrm{mmHg}$ ). Os imigrantes mais jovens apresentaram valores mais baixos de PAS em relação aos mais idosos (140,0 mmHg vs 147,2 mmHg, $p=0,015)$.

\section{Fatores associados à Hipertensão Arterial não contro- lada}

Apresentamos os fatores que se revelaram associados à HTA-TnC, bem como o seu risco atribuível (RA), para imigrantes e nativos, na tabela 3. As diferenças de efeito nos dois grupos não demonstraram interações estatisticamente significativas.

No grupo dos imigrantes, o risco de HTA-TnC quase duplicou no sexo masculino [OR: 1,92; (IC95\%: 1,21 - 3,09); RA: $27,9 \%$ ]. A esse risco também se associou: inexistência de seguro de saúde [2,18; $(1,07-4,61) ; 50,1 \%]$, falta de ajuda para controlar a HTA [2,10; $(1,07-4,31) ; 12,6 \%]$

Tabela 2 - Características da hipertensão arterial e da adesão à terapêutica anti-hipertensiva

\begin{tabular}{|c|c|c|c|}
\hline & $\begin{array}{c}\text { Total } \\
(n=786)\end{array}$ & $\begin{array}{l}\text { Nativos } \\
(n=449)\end{array}$ & $\begin{array}{l}\text { Imigrantes dos PALOP } \\
\qquad(\mathrm{n}=337)\end{array}$ \\
\hline HTA controlada, $n(\%)$ & $365(46,4 \%)$ & $212(47,2 \%)$ & $153(45,4 \%)$ \\
\hline PAS, mmHg, média \pm dp & $141,5 \pm 22,0$ & $141,3 \pm 20,9$ & $141,8 \pm 23,4$ \\
\hline PAD, mmHg, média \pm dp * & $85,7 \pm 12,7$ & $83,6 \pm 11,8$ & $88,4 \pm 13,3$ \\
\hline PAM, mmHg, média \pm dp * & $104,2 \pm 14,5$ & $102,7 \pm 13,4$ & $106,2 \pm 15,5$ \\
\hline \multicolumn{4}{|c|}{ Adesão à Terapêutica anti-hipertensiva, n (\%) } \\
\hline Aderente * & $350(45,0 \%)$ & $234(52,7 \%)$ & $116(34,8 \%)$ \\
\hline Não aderente - não intencional & $277(35,6 \%)$ & $156(35,1 \%)$ & $121(36,3 \%)$ \\
\hline Não aderente - intencional * & $150(19,3 \%)$ & $54(12,2 \%)$ & $96(28,8 \%)$ \\
\hline Não considera a HTA controlada, n (\%) & $150(20,9 \%)$ & $79(19,3 \%)$ & $71(23,1 \%)$ \\
\hline
\end{tabular}

${ }^{*}$ Valor de $p \leq 0,05$, para diferenças nas características entre nativos e imigrantes dos PALOP.

dp: desvio-padrão; HTA: hipertensão arterial; PAD: pressão arterial diastólica; PALOP: Países Africanos de Língua Oficial Portuguesa; PAM: pressão arterial média; PAS: pressão arterial sistólica. 
Tabela 3 - Fatores associados à hipertensão arterial tratada não controlada nos nativos e imigrantes dos PALOP

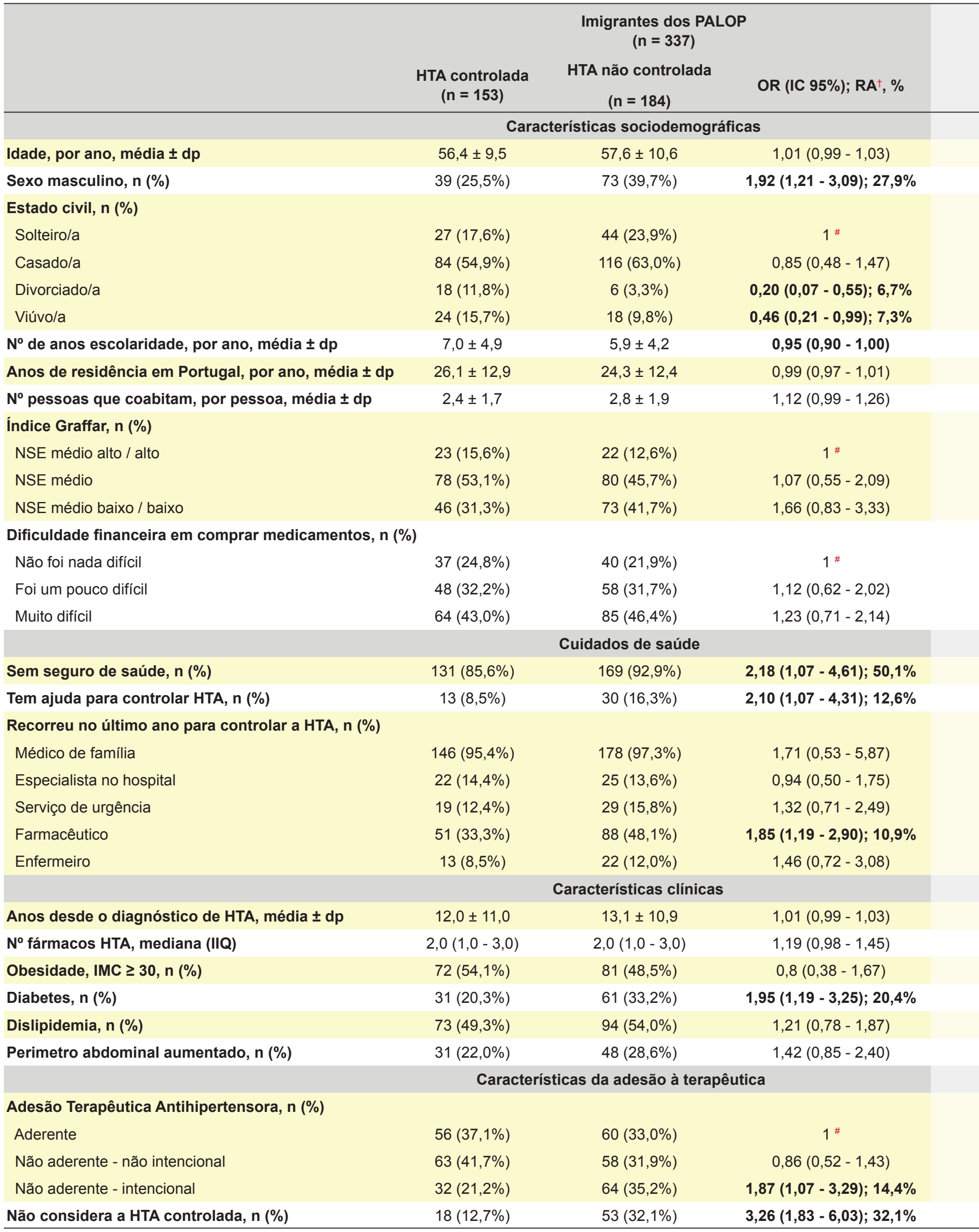

$\mathrm{O}$ odds ratio a negrito têm um valor de $p<0,05$. †: O risco atribuivel é apenas apresentado para as variaveis com associação significativa.

dp: desvio-padrão; HTA: hipertensão arterial; IC: intervalo de confiança; IIQ: intervalo inter-quartílico; IMC: índice de massa corporal; NSE: nível socioeconómico; PALOP: Países Africanos de Língua Oficial Portuguesa; RA: risco atribuível; OR: odds-ratio; \#: classe de referência; * Valor de OR (IC 95\%) Adesão vs Não adesão = 0,85 (0,58 - 1,23). 
Nativos portugueses

( $n=449$ )

HTA controlada

( $n=212)$

HTA não controlada

( $n=237$ )

OR (IC 95\%); RA ${ }^{\dagger}, \%$

\section{Características sociodemográficas}

Idade, por ano, média $\pm \mathrm{dp}$

Sexo masculino, $\mathbf{n}(\%)$

$64,3 \pm 9,1$

$64,2 \pm 9,0$

$1,00(0,98-1,02)$

Estado civil, $\mathbf{n}(\%)$

Solteiro/a

$92(43,4 \%)$

$127(53,6 \%)$

1,51 (1,04 - 2,19); 17,7\%

Casado/a

$8(3,8 \%)$

$11(4,6 \%)$

1 \#

Divorciado/a

$155(73,5 \%)$

$179(75,5 \%)$

$0,84(0,32-2,13)$

$19(9,0 \%)$

$19(8,0 \%)$

$0,73(0,23-2,20)$

Viúvo/a

$\mathbf{N}^{\circ}$ de anos escolaridade, por ano, média $\pm \mathrm{dp}$

$29(13,7 \%)$

$28(11,8 \%)$

$0,70(0,24-1,99)$

$7,1 \pm 4,4$

$6,3 \pm 3,8$

$0,95(0,91-1,00)$

Anos de residência em Portugal, por ano, média \pm dp

$\mathrm{N}^{\circ}$ pessoas que coabitam, por pessoa, média $\pm \mathrm{dp}$

Índice Graffar, $\mathrm{n}$ (\%)

NSE médio alto / alto

NSE médio

NSE médio baixo / baixo

Dificuldade financeira em comprar medicamentos, $\mathbf{n}(\%)$

Não foi nada difícil

Foi um pouco difícil

Muito difícil

$1,4 \pm 1,0$

$1,4 \pm 1,0$

$0,97(0,80-1,17)$

$37(17,9 \%)$

$30(13,4 \%)$

$1^{\#}$

$124(59,9 \%)$

$129(57,6 \%)$

$1,28(0,75-2,21)$

$46(22,2 \%)$

$65(29,0 \%)$

$1,74(0,95-3,23)$

$110(53,9 \%)$

$120(51,5 \%)$

1 \#

$54(26,5 \%)$

$80(34,3 \%)$

$1,36(0,88-2,10)$

$40(19,6 \%)$

$33(14,2 \%)$

$0,76(0,44-1,28)$

\section{Cuidados de saúde}

Sem seguro de saúde, $\mathbf{n}(\%)$

$168(79,2 \%)$

$28(13,4 \%)$

$197(84,2 \%)$

$1,39(0,86-2,27)$

Tem ajuda para controlar HTA, $\mathbf{n}(\%)$

Recorreu no último ano para controlar a HTA, n (\%)

Médico de família

$209(99,1 \%)$

$14(6,6 \%)$

$31(13,2 \%)$

$0,98(0,57-1,71)$

Especialista no hospital

Serviço de urgência

Farmacêutico

$13(6,2 \%)$

$37(17,6 \%)$

$8(3,8 \%)$

Enfermeiro
$13,0 \pm 9,6$

$2,0(1,0-3,0)$

$86(48,3 \%)$

$50(23,6 \%)$

$107(51,4 \%)$

$51(26,8 \%)$
$227(96,6 \%)$

$25(10,6 \%)$

$25(10,6 \%)$

$51(21,9 \%)$

$19(8,1 \%)$

\section{Características clínicas}

$$
14,7 \pm 11,8
$$

$2,0(1,0-3,0)$

$93(47,0 \%)$

$70(29,5 \%)$

$139(60,2 \%)$

$60(28,6 \%)$
$0,27(0,04-1,10)$

$1,67(0,86-3,40)$

$1,81(0,92-3,75)$

$1,31(0,82-2,11)$

1,07 (0,87 - 1,33)

$1,09(0,92-1,3)$

$0,72(0,38-1,36)$

$1,36(0,89-2,08)$

$1,43(0,98-2,09)$

$1,09(0,70-1,69)$
$2,24(0,99-5,54)$

Perimetro abdominal aumentado, n (\%)

Características da adesão à terapêutica

Adesão Terapêutica Antihipertensora, n (\%)
Aderente

Não aderente - intencional

Não considera a HTA controlada, n (\%)
Não aderente - não intencional
$115(54,8 \%)$

$119(50,9 \%)$

$69(32,9 \%)$

$26(12,4 \%)$

$19(9,5 \%)$
$28(12,0 \%)$

$60(28,7 \%)$
$1^{\# *}$

$1,22(0,81-1,83)$

$1,04(0,58$ - 1,89) 
recurso à farmácia para controlo da HTA $[1,85 ;(1,19$ - 2,90); $10,9 \%]$, diabetes $[1,85 ;(1,19-3,25) ; 20,4 \%]$, não adesão à medicação intencional [1,87; $(1,07$ - 3,29); 14,4\%] e perceção (auto reporte) da HTA não estar controlada [3,26; $(1,83$ - 6,03); 32,1\%]. Por cada ano de escolaridade, o risco de HTA-TnC diminuiu 5\% [0,95; $(0,90-1,00)]$. O estado civil divorciado $[0,20 ;(0,07-0,55) ; 6,7 \%]$ ou viúvo $[0,46 ;(0,21-$ $0,99) ; 7,3 \%$ ] esteve associado a menor risco de HTA-TnC. Não encontrámos fatores associados à HTA-TnC que fossem exclusivos dos nativos, apresentando estes um risco de HTA-TnC mais elevado em: sexo masculino [1,51; $(1,04$ - 2,19); 17,7\%], menor escolaridade [0,95; $(0,91-1,00)]$ e perceção (auto reporte) de HTA não controlada [3,84; $(2,23$ - 6,87); 34,9\%]. A adesão à terapêutica não estava associada, de modo estatisticamente significativo, ao controlo da HTA $[0,85(0,58-1,23)]$.

\section{Efeito ajustado de fatores associados à Hipertensão Arterial não controlada}

$\mathrm{Na}$ tabela 4 apresentamos os resultados das análises multivariadas de regressão logística estratificadas para nativos e imigrantes, ajustadas sequencialmente às dimensões sociodemográficas, cuidados de saúde, clínicas e adesão à terapêutica.

No grupo dos imigrantes, o sexo masculino deixou de estar associado à HTA-TnC após ajuste para as variáveis sociodemográficas, utilização de serviços de saúde e clínicas. Ser viúvo $[0,26(0,06-0,99)]$ ou divorciado [0,30 (010$0,86)$ ] manteve-se associado à HTA-TC e os anos de escolaridade deixaram de estar associados à HTA-TC, quando ajustados para todas as características sociodemográficas. Ao nível dos cuidados de saúde, o seguro de saúde ou ter ajuda para controlar a HTA deixaram de estar associados à HTA-TnC quando ajustados às variáveis sociodemográficas. Ter recorrido ao farmacêutico no último ano para controlar a HTA [1,96 (1,10 - 3,50)] permaneceu associado à HTA-TnC quando ajustado para as variáveis sociodemográficas, cuidados de saúde ou clínicas. Nas características clínicas, verificámos que o número de anos desde o diagnóstico de HTA passou a estar associado à HTA-TnC [1,04 $(1,01-1,07)$ ] quando ajustamos aos cuidados de saúde e restantes variáveis clínicas. Por outro lado, o diagnóstico de diabetes perdeu associação com a HTA-TnC quando ajustado às variáveis sociodemográficas, cuidados de saúde ou restantes variáveis clínicas. A não adesão intencional [1,83 (1,01 - 3,35)] nos imigrantes manteve-se associada com a HTA-TnC, mesmo após ajuste para as variáveis sociodemográficas, cuidados de saúde e variáveis clínicas.

A curva ROC para o modelo dos imigrantes apresentou um valor AUC 0,70, o que significa que o modelo estima uma probabilidade superior em $70 \%$ de ter HTA-TnC em relação à HTA-TC (Fig. 2).

No grupo dos nativos, o sexo masculino [OR: 1,97 (IC 95\%: 1,16 - 3,37)] e o número de anos de escolaridade $[0,92(0,86-0,99)]$ mantiveram associação com a HTA-TnC, mesmo após ajuste para as restantes características sociodemográficas, cuidados de saúde e clínicas. Ter con- sultado no último ano o médico de família para controlar a HTA apresentou-se associado à HTA-TC [0,08 (0,01 $0,54)$ ] quando ajustado para variáveis sociodemográficas e manteve-se quando ajustamos para os restantes cuidados de saúde e variáveis clínicas. Ter recorrido no último ano ao serviço de urgência $[3,16(1,32$ - 8,07)] e ao enfermeiro $[3,31(1,09-11,80)]$ para controlar a HTA passou a ser um fator associado à HTA-TnC quando ajustado às variáveis sociodemográficas e manteve-se quando ajustamos aos restantes cuidados de saúde e variáveis clínicas.

A curva ROC para o modelo dos nativos apresentou valor AUC 0,64 (Fig. 2).

\section{DISCUSSÃO}

Tivemos como objetivos comparar a frequência e identificar os fatores associados à HTA-TnC entre hipertensos medicados nativos portugueses (caucasianos) e imigrantes dos PALOP (negros), seguidos nos CSP.

Os resultados obtidos permitem aumentar a compreensão sobre o estado do controlo da HTA nos CSP, quer para nativos portugueses, quer para imigrantes dos PALOP, considerando uma multiplicidade de características e de comportamentos relevantes, com implicações nos cuidados clínicos destes indivíduos. Do ponto de vista metodológico, as observações clínicas são diretas e independentes de consulta ou ida aos CSP. A amostragem foi representativa dos nativos e imigrantes, verificando-se uma excelente taxa de participação em ambos os grupos, com uma sobreamostragem representativa de imigrantes, que aumenta o poder estatístico na comparação com um grupo minoritário. Considerámos o impacto do efeito e da frequência dos fatores em estudo (com estatísticas de RA), modelos específicos para cada grupo e análises multivariadas com ajustes sequenciais, que permitem uma melhor interpretação dos fatores associados em cada grupo.

A taxa de controlo nos hipertensos tratados foi de $46 \%$ no global da amostra, percentagem superior à reportada em Portugal em $2007^{1}$ (28,9\%) e $2010^{2}(30,7 \%)$, o que sugere que o controlo da HTA, quando tratada, tem vindo a aumentar nos últimos anos. Esta evolução confirma-se com os dados obtidos por outros autores em $2011^{38}(47,7 \%)$ e $2014^{39}(55,7 \%)$, e deve-se a uma maior efetividade do tratamento anti-hipertensivo ao nível dos CSP.

Observamos que, apesar do perfil sociodemográfico, clínico e de utilização de serviços de saúde ser díspar entre imigrantes e nativos, estas diferenças não resultaram em níveis de controlo estatisticamente diferentes. Esta observação não está de acordo com a literatura internacional. ${ }^{13-23}$ É possível que os CSP, em Portugal, atuem como fator de redução das desigualdades observáveis entre imigrantes e nativos no que diz respeito ao controlo da HTA. O contexto genético e sociocultural dos imigrantes noutros países pode ser diferente dos PALOP. No entanto, a PAM nos imigrantes foi mais elevada que nos nativos, justificável pelos valores mais elevados da PAD, de acordo com o verificado em vários estudos. ${ }^{20,22}$ Com efeito, sabe-se que, a partir da meia-idade, a PAD tende a diminuir enquanto a PAS tende 


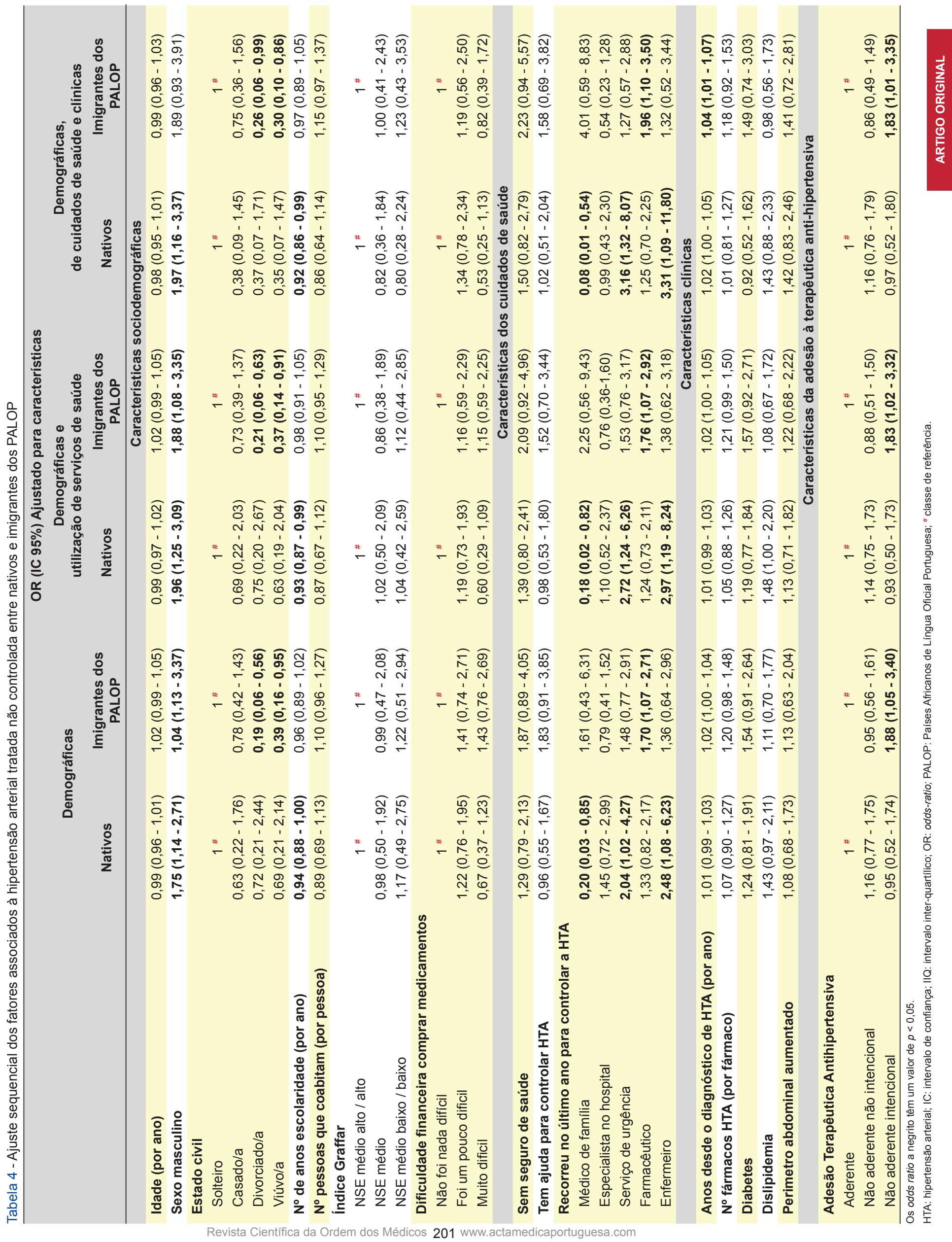






Imigrantes africanos: área sob a curva $\mathrm{ROC}=0,7021$

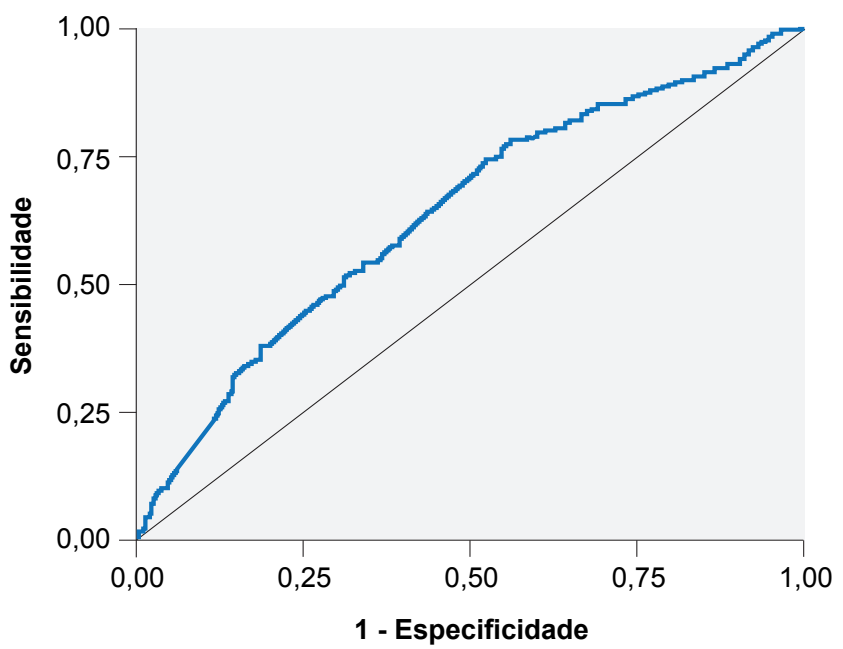

Nativos portugueses: área sob a curva $\mathrm{ROC}=0,6366$

Figura 2 - Curva ROC para hipertensão arterial tratada não controlada nos imigrantes dos PALOP e nos nativos portugueses

a aumentar, ${ }^{40}$ o que se confirmou com os nossos resultados, principalmente nos imigrantes. Sendo estes mais jovens que os nativos, a diferença etária pode justificar a PAD mais elevada, ${ }^{13}$ o que pode ser explicado por alterações funcionais no coração. ${ }^{3}$ Por outro lado, a coexistência de PAD elevada e PAS baixa nos imigrantes com 40-64 anos coloca este subgrupo com maior risco cardiovascular. ${ }^{40}$

Poucos fatores estavam associados à HTA-TC nos nativos, salientando-se o sexo masculino e o menor grau de escolaridade, fatores já definidos noutros estudos portugueses..$^{1,24-26}$ Espiga de Macedo et $\mathrm{al}^{1}$ apresentaram como hipótese que as mulheres irão mais vezes ao médico e Pereira et al. ${ }^{25}$ sugeriram que a associação negativa com os anos de escolaridade se deverá a um confundimento com a idade, mas no nosso estudo a evidência é que o sexo masculino e o menor grau de escolaridade mantêm-se associados à HTA-TnC, independentemente da utilização dos cuidados de saúde ou da idade.

Nos imigrantes, o sexo masculino deixou de estar relacionado com a HTA-TC quando ajustamos para as características clínicas. Alguns estudos sugerem que o sexo é um fator de confundimento, ${ }^{20}$ sendo a toma de mais medicação a responsável por a HTA estar mais controlada. ${ }^{16,20}$ Contudo, os nossos dados não revelaram diferenças significativas no número médio de medicamentos tomados entre controlo e não controlo da HTA, tal como no estudo de Oliveira-Martins et al, ${ }^{38}$ mas sim com os anos de duração da doença.

O estado civil divorciado ou viúvo, nos imigrantes, parece associado a menor risco de HTA-TnC, independentemente das restantes variáveis. Até ao momento, o estado civil não tem sido considerado um fator relevante nos estudos sobre o controlo da HTA. Note-se que "ter ajuda para controlar a HTA" deixou de estar associado ao controlo da HTA nos imigrantes após ajuste para características sociodemográficas, pelo que esta variável poderá encontrar-se confundida pelo estado civil nesta população.

A relação muito forte entre 'não considerar a HTA con- trolada' e a HTA-TnC significa que muitos hipertensos têm conhecimento do não controlo da sua HTA, talvez por serem informados por profissionais de saúde ou realizarem automedições. Isto sugere que ter a HTA-TnC não se deve, necessariamente, à falta de seguimento ou informação sobre o seu estado de controlo. Nos imigrantes, a HTA-TnC associa-se também à não adesão intencional à medicação anti-hipertensiva. Estes aspetos salientam que é crucial a capacitação e empoderamento do doente crónico para a gestão do controlo da sua doença. É fundamental perceber se o controlo da HTA poderá ser afetado, e como, pela perceção e atitudes dos hipertensos sobre a sua doença, o que deverá ser alvo de um estudo específico.

Os nativos são mais aderentes à medicação, mas não observámos uma associação entre adesão e controlo. Isto sugere que, nos hipertensos tratados seguidos nos CSP, o efeito da não adesão pode ser compensado, ao longo do acompanhamento, por elevações da carga terapêutica, resultando em níveis semelhantes de controlo. Contudo, o número de fármacos anti-hipertensivos não surgiu associado à HTA-TnC em nenhum dos grupos, apesar de diversos estudos sugerirem que os africanos necessitam de um tratamento mais intensivo que os caucasianos para se conseguir controlar a HTA. ${ }^{20-22}$ Este dado apoia a hipótese do controlo tensional nos africanos depender mais da qualidade dos fármacos (classes) do que da sua quantidade (tomas diárias). ${ }^{14,24}$ Estas hipóteses devem ser confirmadas em estudos desenhados com esse objetivo.

Nos imigrantes, o controlo da HTA era superior à adesão à medicação, o que se deveu ao facto da sua maioria tomar a medicação referindo apenas não dar atenção à hora da sua toma (não adesão não intencional). A não adesão à medicação intencional mostrou encontrar-se associada ao não-controlo tensional, o que está de acordo com outros estudos. ${ }^{13,16,22}$ Esta opção resulta de muitos imigrantes declararem interromper a medicação por deixarem acabar os medicamentos, por seguirem indicações que não foram dadas pelo médico dado se sentirem melhor ou pior. 
Este tipo de comportamento pode estar relacionado com crenças sobre o papel da medicação, ${ }^{33}$ cuja associação deverá ser alvo de um estudo específico.

A idade, o nível socioeconómico e a dificuldade financeira em comprar medicamentos não estavam associados à HTA-TnC, o que é consistente com outros estudos ${ }^{17,20,38}$ e sugere que, em Portugal, mesmo pessoas com baixos recursos têm acesso aos medicamentos. Nos imigrantes, o risco de HTA-TnC aumentou 4\% por cada ano após o diagnóstico de HTA, independentemente da idade e das variáveis clínicas. Num estudo feito no Porto, ${ }^{25}$ a idade precoce do diagnóstico de HTA era um fator associado à HTA-TnC, o que provavelmente estará relacionado com a gravidade da doença.

A obesidade, de que sofriam quase $50 \%$ dos hipertensos, não se relacionou com a HTA-TnC, e nos imigrantes o diagnóstico de diabetes perdeu associação quando ajustado para as variáveis sociodemográficas. Estes resultados são idênticos aos de outras investigações. Por exemplo, Agyemang e Bhopal ${ }^{20}$ verificaram que a obesidade influenciava o controlo da HTA apenas nas mulheres, concluindo que o Índice de Massa Corporal é um fator de confundimento. Num estudo realizado em Portugal, ${ }^{25}$ verificou-se que a diabetes apenas se associa ao conhecimento sobre a HTA nas mulheres mas não ao seu tratamento e controlo.

Ao nível da utilização dos cuidados de saúde, recorrer ao médico de família para controlar a HTA esteve fortemente associado ao controlo da HTA nos nativos, o que está de acordo com Oliveira-Martins et al. ${ }^{38}$ Os imigrantes recorreram mais a outros profissionais de saúde para controlar a sua HTA, apesar de serem utilizadores dos CSP e estarem integrados (viviam em Portugal, em média, há 25 anos). A ida à farmácia para controlar os valores tensionais revelou estar associada a menor controlo dos imigrantes hipertensos tratados. Possivelmente este recurso pode ser preferido por razões de tempo de espera ${ }^{12}$ e/ou custos ${ }^{9}$ inferiores à ida ao médico de família. Um estudo efetuado em Lisboa com imigrantes com mais de 15 anos, maioritariamente dos PALOP, verificou que estes utilizavam pouco os CSP devido a: falta de documentos legais, longo tempo de espera, mau atendimento, falta de médico de família, desadequação do período de funcionamento, pouca consciência da importância dos serviços de saúde e, inclusive, a perceção de que a saúde não é importante recorrendo aos serviços de saúde apenas em caso de extrema necessidade. ${ }^{41}$ Ajustando às características sociodemográficas, os hipertensos não controlados nativos tenderam a recorrer com maior probabilidade ao Serviço de Urgência e ao Enfermeiro enquanto os imigrantes tenderam a recorrer mais ao farmacêutico. Ambos recorreram ao médico de família com o propósito de controlar a HTA, mas com um efeito apenas percetível nos nativos. Isto salienta a necessidade de incrementar a acessibilidade aos CSP para acomodar situações de falta de controlo desta doença crónica e meIhorar a relação médico-doente de modo a possibilitar uma utilização adequada dos CSP, sem negligenciar o papel de outras estruturas e profissionais de saúde no controlo da
HTA.

Este estudo tem algumas limitações. A análise transversal não permite atribuir causalidades ou definir a direção de um efeito. A população alvo foi utentes dos CSP na área da Grande Lisboa, não se podendo extrapolar os dados a nível nacional. O valor de curva ROC da análise multivariada dos nativos sugere pouca capacidade em concluir qual a relação entre controlo da HTA e as restantes características ${ }^{37}$ Seria importante incluir outras variáveis, como as crenças de saúde. Nos imigrantes, a área obtida possui capacidade discriminatória aceitável. ${ }^{37}$

\section{CONCLUSÕES}

No total, dos hipertensos tratados nos CSP, $46 \%$ tinham a hipertensão controlada. Este indicador sugere que existe um potencial de melhoria e confirma o papel essencial do médico de família no controlo do fator de risco com maior impacto na prevenção e controlo das doenças crónicas - a hipertensão arterial.

Uma grande parte dos participantes tinha conhecimento da falta de controlo tensional e várias características revelaram-se associadas ao menor controlo dos hipertensos tratados: nos nativos, o sexo masculino e o menor grau de escolaridade; nos imigrantes, a maior duração da HTA e a não adesão intencional à terapêutica anti-hipertensiva.

Os grupos de hipertensos nativos portugueses e imigrantes dos PALOP diferenciaram-se no efeito da consulta do médico de família, bem como no recurso a outros serviços e profissionais de saúde, para controlar a HTA. No entanto, estas diferenças não resultaram em níveis de controlo estatisticamente diferentes. Por outro lado, concluímos que os imigrantes mais jovens são um subgrupo com maior risco cardiovascular.

Os resultados deste estudo podem ter impacto na definição de estratégias e recomendações para o controlo da hipertensão nos CSP na medida em que sugerem abordagens diferenciadas para algumas especificidades étnicas (biológicas, comportamentais e culturais).

\section{AGRADECIMENTOS}

Os autores gostariam de agradecer aos participantes no estudo, às Unidades de Saúde da Região de Lisboa e Vale do Tejo, aos profissionais envolvidos, e a colaboração de todos os bolseiros e estagiários de investigação.

\section{CONFIDENCIALIDADE DOS DADOS}

Os autores declaram ter seguido os protocolos do seu centro de trabalho acerca da publicação dos dados de doentes.

\section{PROTECÇÃO DE PESSOAS E ANIMAIS}

Os autores declaram que os procedimentos seguidos estavam de acordo com os regulamentos estabelecidos pelos responsáveis da Comissão de Investigação Clínica e Ética e de acordo com a Declaração de Helsínquia da Associação Médica Mundial. 


\section{CONFLITO DE INTERESSES}

Os autores declaram não ter nenhum conflito de interesses relativamente ao presente artigo.

\section{FONTES DE FINANCIAMENTO}

O estudo DIMATCH-HTA teve o apoio científico da Associação Portuguesa de Medicina Geral e Familiar, foi financiado pela Fundação para a Ciência e Tecnologia (PTDC/SAU-ESA/103511/2008) e pelos Laboratórios Delta, e foi premiado pela Fundação AstraZeneca.

\section{REFERÊNCIAS}

1. Macedo ME, Lima MJ, Silva AO, Alcântara P, Ramalhinho V, Carmona J. Prevalência, Conhecimento, Tratamento e Controlo da Hipertensão em Portugal. Estudo PAP. Rev Port Cardiol. 2007;26:21-39.

2. Polónia J, Mesquita-Bastos J, Pessanha P, Bertoquini S, Martins L, Silva JA et al. Estratificação do Risco Cardiovascular global de doentes hipertensos seguidos nos cuidados de saúde primários ou hospitalares segundo as orientações ESH/ESC 2007. Rev Port Cardiol. 2010;29:1685-96.

3. Mendes R, Themudo Barata JL. Envelhecimento e pressão arterial. Acta Med Port. 2008;21:193-8.

4. Psaty BM, Lumley T, Furberg CD, Schellenbaum G, Pahor M, Alderman $\mathrm{MH}$ et al. Health outcomes associated with various antihypertensive therapies used as first-line agents: a network meta-analysis. JAMA. 2003;289:2534-44.

5. Direcção-Geral de Saúde. Programa Nacional de Prevenção e Controlo das Doenças Cardiovasculares. Despacho n. ${ }^{0} 16415 / 2003$, DR, II Série, de 22 de Agosto, page 12. [Consultado 2015 Ago 28]. Disponível em http://www.dgs.pt/.

6. Direcção-Geral de Saúde. Programa Nacional para as Doenças Cardiovasculares: Orientações Programáticas. Documento publicado a 5 de setembro de 2012 [Consultado 2015 Ago 28]. Disponível em: http:// www.dgs.pt/programas-de-saude-prioritarios.aspx

7. Estrela J, Machado R, Bento AR, Martins LA, Sousa P. Relatório de imigração, fronteiras e asilo 2011. Serviços de Estrangeiros e Fronteiras/ Departamento de Planeamento e Formação. 2012.

8. Mancia G, Fagard R, Narkiewicz K, Redon J, Zanchetti A, Böhm M et al. ESH-ESC Task Force for the Management of Arterial Hypertension. 2013 ESH-ESC Guidelines for the Management of Arterial Hypertension. Eur Heart J. 2013;34:2159-219.

9. Estrela P. A saúde dos imigrantes em Portugal. Rev Port Clin Geral. 2009;25:45-55.

10. Geronimus AT, Bound J, Keene D, Hicken M. Black-White differences in age trajectories of hypertension prevalence among adult women and men, 1999-2002. Ethn Dis. 2007;17:40-8.

11. Brown MJ. Hypertension and ethnic group. BMJ. 2006;332:833-6.

12. Dias SF, Severo M, Barros $H$. Determinants of health care utilization by immigrants in Portugal. BMC Health Serv Res. 2008;8:207.

13. Spritzer N. Epidemiologia da hipertensão arterial sistémica. Medicina (Ribeirão Preto), 1996;29:199-213.

14. Agyemang C, Kieft S, Snijder MB, Beune EJ, van den Born BJ, Brewster LM et al. Hypertension control in a large multi-ethnic cohort in Amsterdam, the Netherlands: the HELIUS study. Int $\mathrm{J}$ Cardiol, 2015;183:180-9.

15. Gus I, Harzheim E, Zaslavsky C, Medina C, Gus M. Prevalência, reconhecimento e controle da hipertensão arterial sistémica no Estado do Rio Grande do Sul. Arq Brasil Cardiol. 2004;83:424-8.

16. Jackson JH, Bramley TJ, Chiang TH, Jhaveri V, Frech F. Determinants of uncontrolled hypertension in an African-American population. Ethn Dis. 2002;12:53-7.

17. Shelley D, Tseng $T$, Andrews $H$, Ravenell J, Wu D, Ferrari $P$ et al. Predictors of blood pressure control among hypertensives in community health centers. Am J Hypertens. 2011;24:1318-23.

18. Ferdinand KC. Hypertension in minority populations. J Clin Hypertens, 2006;8:365-8.

19. Downie DL, Schmid D, Plescia MG, Huston SL, Bostrom S, Yow A et al. Racial disparities in blood pressure control and treatment differences in a Medicaid population, North Carolina, 2005-2006. Prev Chronic Dis. 2011;8:A55.

20. Agyemang $C$, Bhopal R. Is the blood pressure of people from African origin adults in the UK higher or lower than that in European origin white people? A review of cross-sectional data. J Hum Hypertens. 2003;17:523-34.

21. Decoste M, Vanobberghen R, Borgermans L, Devroey D. Uncontrolled hypertension among black Africans in the city of Brussels: a case-control

study. Eur Rev Med Pharmacol Sci. 2013;17:886-94.

22. Agyemang C, Bindraban N, Mairuhu G, Montfrans Gv, Koopmans R, Stronks $\mathrm{K}$ et al. Prevalence, awareneness, treatment, and control of hypertension among African Surinamese, South Asian and white Dutch in Amsterdam, the Netherlands - The SUNSET study. J Hypertens. 2005;23:1971-7.

23. Agyemang C, van Valkengoed I, Koopmans R, Stronks K. Factors associated with hypertension awareness, treatment and control among ethnic groups in Amsterdam The Netherlands: The SUNSET study. J Hum Hypertens. 2006;20:874-81.

24. Cortez-Dias N, Martins S, Belo A, Fiuza M. Prevalência e padrões de tratamento da hipertensão arterial nos Cuidados de Saúde Primários em Portugal. Resultados do estudo VALSIM. Rev Port Cardiol. 2009;28:499-523

25. Pereira M, Azevedo A, Barros H. Determinants of awareness, treatment and control of hypertension in a Portugueses population. Rev Port Cardiol. 2010;29:1779-92.

26. Perdigão C, Rocha E, Duarte JS, Santos A, Macedo A. Prevalência caracterização e distribuição dos principais factores de risco cardiovascular em Portugal. Uma análise do estudo AMÁLIA. Rev Port Cardiol. 2011;30:393-432.

27. Morgado M, Rolo S, Macedo AF, Pereira L, Castelo-Branco M. Predictors of uncontrolled hypertension and antihypertensive medication nonadherence. J Cardiovasc Dis Res. 2010;1:196-202.

28. DIMATCH-HTA: Impacto da Adesão e da Mudança Terapêutica no Controlo da Tensão Arterial. [Consultado 2015 Ago 28]. Disponível em: http://www.uepid.org/projectos-dimatch.

29. Mancia G, De Backer G, Dominiczak A, Cifkova R, Fagard R, Germano $G$ et al. ESH-ESC Task Force on the Management of Arterial Hypertension. 2007 ESH-ESC Practice Guidelines for the Management of Arterial Hypertension. J Hypertens. 2007;25:1751-62.

30. Salvi P. Mean Arterial Pressure. Em: Pulse Waves: How vascular hemodynamics affects blood pressure. Springer. 2012;XII:3-7.

31. Graffar M. Une méthode de classification sociale d'echantillons de population. Courier. 1956;6:455- 9.

32. Delgado $A B$, Lima ML. Contributo para a validação concorrente de uma medida de adesão aos tratamentos. Psic Saúde Doenças. 2001;2:81100

33. Unni EJ, Farris KB. Unintentional non-adherence and belief in medicines in older adults. Patient Educ Couns. 2011;83:265-8.

34. Wroe AL. Intentional and Unintentional Nonadherence: a study of decision making. J Behav Med. 2002;25:355-72.

35. Lehane E, McCarthy G. Intentional and unintentional medication nonadherence: a comprehensive framework for clinical research and practice? A discussion paper. Int J Nurs Stud. 2007;44:1468-77.

36. Society, the Individual and Medicine. Attributable Risk and Population Attributable Risk (PAR) Measures. Ottawa, Canada University. [Consultado em 2015 Ago 28]. Disponível em: http://www.med.uottawa. ca/sim/data/PAR_e.htm.

37. Hosmer Jr DW, Lemeshow S, Sturdivant RX. Applied logistic regression. Berlin: Wiley; 2013.

38. Oliveira-Martins S, Oliveira T, Gomes JJ, Caramona M, Cabrita J. Factores associados à hipertensão arterial noss utentes de farmácias em Portugal. Rev Saúde Pública. 2011;45:136-44.

39. Polonia J, Martins L, Pinto F, Nazaré J. Prevalence, awareness, treatment and control of hypertension and salt intake in Portugal: changes over a decade. The PHYSA study. J Hypertens. 2014;32:121121.

40. Rocha E. Influência da pressão arterial sistólica e pressão arteria diastólica na repercussão nos órgãos alvo. Rev Factores Risco. 2013;28:16-9

41. Gonçalves A, Dias S, Luck M, Fernandes MJ, Cabral J. Acesso aos cuidados de saúde de comunidades migrantes: problemas e perspectivas de intervenção. Rev Port Saúde Pública. 2003;21:55-64. 